\title{
Reform and Practice of the Cultivation of Engineering Quality and Creative Ability through the Specialty Experiment Teaching
}

\author{
Zaibo Li \\ School of Civil Engineering \\ Shaoguan University \\ Shaoguan, People’s Republic of China
}

\author{
Yongping Zhang \\ School of Foreign Languages \\ Shaoguan University \\ Shaoguan, People’s Republic of China
}

\begin{abstract}
In order to meet the requirements for the chemical professional personnel, teaching reform and practice in the cultivation of engineering quality and creative ability was performed. The reform methods included presenting engineering views during the teaching course, opening laboratory for students, establishing comprehensive experiments and building showing the area of instrument installation. The practice proves that the reform effect is prominent and the students' engineering ability and creative ability are improved remarkably.
\end{abstract}

Keywords-Engineering Quality; Creative Ability; Chemical Engineering Principle; Experiment Teaching

\section{INTRODUCTION}

In higher engineering education, the perfect knowledge system framework includes basic scientific knowledge, engineering knowledge, engineering technology and equipment knowledge. From natural science to engineering technology, there must be an intermediate level of knowledge, and the education of this intermediate level of knowledge is undertaken through the basic course of technology [1]. The principle of chemical engineering is a basic course of technology, which plays a bridge between science and engineering in the knowledge education plan of chemical engineering students. The main purpose of engineering course teaching is to combine theory with practice demonstration education for engineering students, cultivate students' engineering concept, engineering consciousness, ability to analyze engineering problems and solve engineering problems, and cultivate engineering professional quality [2]. This kind of training is not only reflected in the teaching process of how to obtain engineering theories through basic scientific theories but also reflected in the teaching process and training process of how to apply engineering ideas and engineering theories and methods to solve various engineering problems.

The development of the chemical industry and society puts forward new requirements for the cultivation of higher education talents [3]. The development and application of new technologies and products need a large number of talents with high engineering quality, high skills and innovative ability while experimental teaching is an effective way of cultivating students' engineering practice ability and innovation ability. As a local undergraduate institution, our general objectiveorientation is making experimental teaching closely surround

This research was financially supported by the Foundation of Shaoguan University (SYJY20171811). the idea that construction and social regional economic development integrate closely with distinctive features and outstanding advantages, and becoming an influential application-oriented local university in similar colleges and universities [4]. This objective-orientation is suitable for the demand of social development and attaches importance to enhance the cultivation of students' engineering ability which has become the key issue of current educational reform. So in this paper, our school's reform and practice of cultivating students' engineering quality and innovation ability in the teaching of Chemical Engineering Principle experiments are discussed.

\section{The FunCtion AND CURRENT TEACHING SituAtion OF CHEMICAL ENGINEERING PRINCIPLE EXPERIMENT COURSES}

The Chemical Engineering Principle is a basic technological course in practice, and it uses the basic theory of natural science to analyze and deal with the physical process in the chemical production process, while the experiment of Chemical Engineering Principle is an engineering experiment course, which is different from the basic experiment. The engineering experiment plays a transitional role in basic courses and specialized courses, and it is a bridge from chemistry to chemical engineering, from science to engineering courses. By the experimental teaching of Chemical Engineering Principle students' understanding of basic chemical engineering principles that they learned from the classes of theory teaching has improved, and the deficiency of in-class theory teaching has been remedied and students' perceptual knowledge has been increased by the training of experiment skill and research methods; meanwhile it can cultivate students' engineering concept and can give students the training of understanding the engineering importance of experimental results for designing production equipment and solving practical problems [5]. In this way, students can draw conclusions step by step by experimental data and propose their own opinions, and furthermore, students' sense of innovation, and the ability of synthetically utilizing theoretical knowledge to analyze and solve practical problems will be enhanced.

Chemical engineering principle is a chemical engineering professional foundation courses and professional backbone course. Through the course of learning, the students understand the task characteristics and the basic content of principles of 
chemical engineering course, understand the chemical process and its basic unit, master the basic concept of fluid conveying, dry, distillation, extraction and diathermancy, master the basic equation of the hydrostatics, Bernoulli equation, and the fluid conveying basic calculation, the basic calculation of absorption, dry and distillation. The learning of this course improves students' ability to analyze and solve problems in fluid transport, heat transfer and mass transfer processes.

The rapid development of modern chemical technology gives a higher demand for Chemical engineering professionals. In the current experiment teaching mode of chemical engineering principle, the problems and aspects that are inappropriate to the demand for talents. Details are as follows: on the one hand, students usually learn passively and in this traditional teaching mode the students' interests for experiment can't be stimulated, which has a strong impact on the cultivation of students' capacity for independent operation, innovation and engineering quality; on the other hand, the teaching contents are unitary and because of the limitation of current experimental condition only several classical operation experiments are set up in the lab, which can't reflect the development of modern chemical technology. Meanwhile, the teaching resources of lab in local universities are relatively scanty, so in order to complete the teaching tasks students are divided into groups in the experimental process in which each student only takes charge of certain experimental operation. Such case results in students' inadequate realization for the mutual effect among parameters which will affect the overall experiment result, and students can complete experiments just by the operation steps on the lab handouts without mindful work. From this kind of experiment, the real knowledge students acquired is very limited [6].

\section{Cultivating STUdents’ ENGINEERING QUALITY AND INNOVATION ABILITY IN EXPERIMENT TEACHING}

Combining with the teachers' teaching, scientific research and experiences of their engineering practice, experiment teaching centers on cultivating students' operational ability, engineering concept and spirit of innovation, thus the ideas of teaching reforms that focus on cultivating practical ability and innovative competence will be permeated into experiment teaching.

1. The Osmosis of Engineering Views in Experiment Teaching. The unit operations of chemical engineering are the research contents of the Chemical Engineering Principle. In the learning of units' operation, equipment information and conventional engineering calculation method, the on-the-spot technicians' engineering concepts, knowledge and engineering analysis methods are combined with the learning of units' operations so that they can be compared systematically, introduced thoroughly, emphasized repeatedly and understood deeply. Before the experimental lessons, the independent learning periods will be scheduled to explain the experimental facilities in the laboratory and their technical principles, and to analyze the measuring methods, theory, ad parameters that should be collected in the experiments, thus students can think about engineering problems actively before they enter into laboratory. The basic teaching thought of each unit's operation is as follows: from the introduction of practical problems in industrial production the accuracy of physical conception will be emphasized, and the basic operation and theory of each unit will be raised in essence, and then more examples in production will be used to introduce the core theory and application of formulas; emphasize the rules that describe process and analyze the elements that affect process completely so that the students' capabilities in analysis and solving problems will be cultivated; in the process of operation experiment, students will be guided to think actively about the reasons of collecting the required parameters of the current experiment, predict the possible results of the experiment, analyze the importance of the operating rules in experimental facilities and the points for attention in experimental process, etc.; in the choice of examples and exercises, the contents about finding pictures and graphs, selecting formulas reasonably should be added thus students can gain the training about consulting engineering manual and technical materials repeatedly.

2. Integrating the current experimental resources, establishing comprehensive experiment and cultivating students' comprehensive abilities in analyzing problems. For example, in the experiment of conveying equipment for fluid substances, four experiments can be an established-Reynolds experiment, curve test of centrifugal pump, the experiment of calibrating flow meter, and experiment of flow resistance testing. By the experimental facility of "hydrodynamics", the four mentioned experiments that belong to the same unit operations can be integrated and thus students can understand the technological process of hydromechanics experiment on the whole, which will cultivate students' comprehensive ability to analyze problems and be suitable for the learning requirements of students with different levels and interests. At the same time, in the experimental teaching task-driven teaching methods should be adopted to make students learn while operating, so that they can learn during experimental operation, experience the practical application of theory and achieve the teaching objective of learning in order to practice.

3. Carrying out the transformation and development of the experimental facilities of the Chemical Engineering Principle gradually, developing and improving students' engineering quality in practice. In the lab there are some junked experimental facilities of chemical engineering principle that are purchased by college 20 years ago, and this project aims to organize students in groups to make the surveying and mapping and restructuring to this junked experimental facilities with the learned knowledge of engineering drawing, machine design foundation, physics, chemistry and the basic theory of Chemical Engineering Principle Course. The teaching function of the old experimental facilities will be renewed and the experimental projects will be added while the students' ability of engineering application will be trained and improved.

4. Opening up the lab of chemical engineering principle, combining with students' extracurricular scientific and technological activities, and attaching importance to teachers' effect of their words and deeds in classes. The lab opens up so according to their time students can make the appointment time to enter the lab for the experiment. When guide students, teachers should pay attention to the basic skills, education of experimental attitude, the cultivation of the down-to-earth 
attitude in scientific research. Students apply for conducting the experiment and teachers put forward the experimental requirements. The experimental adviser should examine the experimental schemes which were formulated by students and after that students complete the experiment independently, which emphasizes students' independent experimental ability and the cultivation of independent work and form a good habit of scientific research. Combined with extracurricular scientific and technological activities this way can expand experimental learning and research ability, and improve students' innovation capability by extra-curricular groups. Depending on the experimental platform of chemical engineering principle, extracurricular groups of chemical engineering principle was formed in a chemical engineering specialty, thus the discussion about the technology of chemical engineering principle will be launched and students can be organized in a planned way to take part in various kinds of science and technology competitions.

5. Establishing chemical instrumentation, facility and accessory exhibition area, developing relevant materials about multimedia chemistry and demonstrating. By taking the opportunities of cooperation with enterprises or the internship into them to collecting, various kinds of chemical pump, valve, pipe fitting, flow meter, draught fan, stirrer device, heatexchanger components, padding, column plate, membrane module and meters have been collected widely; or the available components of junked experimental devices have been torn down to help students observe the real objects, further enhance their perceptual knowledge for industrial production equipment, and realize directly the engineering quality of chemical engineering principle. There are other ways such as researching and developing the relevant chemical materials, simulating and demonstrating the relevant process of the experimental phenomenon and unusual operation or phenomena that are difficult to notice in the experiment. For example, when the centrifugal pump cavitation takes place actually the centrifugal pump impeller can be damaged easily, but the 3D animation can simulate the centrifugal pump cavitation, which can not only let students see the cavitation phenomenon but also avoid damaging experimental facilities. Another example is that fluid flows in pipeline and the condition of gas-liquid contacting in rectifying tower can't be seen visually, but by the courseware the experimental phenomena that can't be observed because of the limitation of experiment condition or the fragile experimental facilities can be visual, so in this way the experiment change rules can be observed clearly and the complex principles can be presented vividly for students. Meanwhile, in this way, students can gain more perceptual knowledge, and the combination of theory and practice will be enhanced, which is beneficial to the cultivation of students' abilities in analyzing and solving problems.

\section{CONCLUSION}

As a specialized fundamental course the experiment of chemical engineering principle plays an important role in the curriculum system of chemical engineering majors, and functions as a link in the formation of knowledge structure for professionals. The relevant measures of teaching reform have been carried out in the experimental teaching of our college, and the curriculum reform and teaching have received students' wide recognition. Meanwhile, students speak highly of these reforms and they generally believe that Chemical Engineering Principle and its experiment are the best, most impressive, most harvested and most unforgettable courses they learned. Each year the graduates who choose Chemical Engineering Principle as the course of their postgraduate entrance examination have been admitted by key universities with excellent marks. When students graduated and joined in work, students from our college have been regarded as good adaptability and strong practical ability by employers. The cultivation and training content that students have received during school help graduates better adapt to the practical requirements in engineering. The teaching reforms have gained the first prize for teaching research. The practice has proven that the teaching reform of the Chemical Engineering Principle experiment plays an important role in improving the comprehensive abilities for undergraduates of chemical major, and lays an important foundation for cultivating students' engineering idea, application concept, and abilities to analyze and solve practical engineering problems.

\section{REFERENCES}

[1] Ding Yuansheng, Lu Fei. Establishment of Practice Teaching System Based on Plan of Educating and Training Outstanding Engineers for the Applied Chemistry in the Local Colleges, Guangdong Chemical Industry, 2016, 43(01), 165-165+171.(In Chinese)

[2] Deng-xiang, ZHANG Yun, YING Hui-juan. Teaching Exploration for Experiment of Chemical Engineering Principle under Background of Engineering, Education Teaching Forum, 2018, (43), 265-267. (In Chinese)

[3] Zhou Yanjun, Zhang Qijian, Zhao Yonghua. Strengthening Teaching Innovation in Principles of Chemical Engineering Based on Cultivating Students' Engineering Quality, China Modern Educational Equipment, 2014, (23), 44-45. (In Chinese)

[4] Li Fenggang, Ju Caixia, Dong Kai. Discussion on Experimental Teaching Mode of Chemical Engineering Principle, Shandong Chemical Industry, 2019, 48(11), 157-157+159. (In Chinese)

[5] Wang Yunhua, Wang Heyun. Discussion on Construction and Function of Engineering Contextual Language in the Principles of Chemical Engineering Teaching, Higher Education in Chemical Engineering, 2015 32(06), 89-93+96. (In Chinese)

[6] Chen Yong. Engineering Literacy Education in the Teaching of Principles of Chemical Engineering of the Applied University, Higher Education in Chemical Engineering, 2017, 34(06), 75-78. (In Chinese) 